\title{
Transplant coronary heart disease: challenges and solutions
}

This article was published in the following Dove Press journal:

Transplant Research and Risk Management

II December 2014

Number of times this article has been viewed

\author{
Jacob C Jentzer' \\ Gavin W Hickey' \\ Sameer J Khandhar ${ }^{2,3}$ \\ 'Heart and Vascular Institute, \\ University of Pittsburgh Medical \\ Center, Pittsburgh, PA, USA; ${ }^{2}$ Perelman \\ School of Medicine, University \\ of Pennsylvania, Philadelphia, PA, \\ USA; ${ }^{3}$ Heart and Vascular Institute, \\ Penn-Presbyterian Medical Center, \\ Philadelphia, PA, USA
}

\begin{abstract}
Cardiac allograft vasculopathy (CAV) remains one of the leading causes of death and graft failure after heart transplantation. A variety of causes, including donor heart characteristics, recipient risk factors, and immune-mediated influences, are associated with developing CAV. In this review, we will focus on the pathophysiology of developing CAV and various methods to screen for this condition. The pathogenesis of CAV likely involves repeated injuries to the endothelium from a variety of factors such as cellular-mediated rejection, and alloimmune factors, including antibody-mediated injury, ischemia-reperfusion injury at time of transplant, cytomegalovirus infections, immunosuppression medications, systemic inflammation, and traditional atherosclerosis risk factors. Patients with significant CAV are often asymptomatic, and therefore early detection by routine screening prior to graft dysfunction is crucial. There are a variety of invasive, noninvasive, and blood tests that have been studied as screening methods, and we will discuss the role of each of these in this review article. Although some treatment regimens have been established for $\mathrm{CAV}$, this is an area where further studies and research are necessary.
\end{abstract}

Keywords: cardiac allograft vasculopathy, orthotopic heart transplantation, intra-vascular imaging

\section{Introduction}

Cardiac allograft vasculopathy (CAV) is widely considered the "Achilles heel" of heart transplantation. ${ }^{1}$ Early posttransplant survival has improved, but median survival remains only 10.4 years. CAV is the leading cause of graft failure and the second leading cause of death in patients living more than 3 years posttransplant. ${ }^{2} \mathrm{CAV}$ is a common clinical problem, occurring in $>50 \%$ of orthotopic heart transplant (OHT) patients by 10 years, with significant associated morbidity and long-term mortality.

Although malignancy has become the number one cause of mortality late after transplantation, CAV accounts for $10 \%-14 \%$ of deaths beyond the first year after transplant..$^{3-6}$ Death due to CAV typically occurs as the result of graft failure, arrhythmias, or sudden cardiac death. ${ }^{3}$ Early-onset CAV occurs within the first year after OHT and is especially associated with worse long-term outcomes. ${ }^{3,4}$ Screening and diagnosing CAV presents a difficult clinical dilemma. Most patients with CAV are asymptomatic because of cardiac sensory denervation, which prevents the development of typical angina symptoms. Therefore, there is increasing interest in screening patients for subclinical disease, but no consensus on the ideal method has been reached. Screening has been performed with conventional tests such as
Correspondence: Sameer J Khandhar

Heart and Vascular Institute,

Penn-Presbyterian Medical Center, 4th Floor PHI, 5I North 39th Street, Philadelphia, PA 19104, USA

Tel +l $215662917 \mid$

Fax + I 2152434637

Email sameer.khandhar@uphs.upenn.edu
Dovepress

http://dx.doi.org/10.21 47//TRRM.S50846
Transplant Research and Risk Management 2014:6 I I7-127

(c) (i) (\$) $\odot 2014$ Jenter et al. This work is published by Dove Medical Press Limited, and licensed under Creative Commons Attribution - Non Commercial (unported, v3.0) 
stress testing, coronary angiography, and intravascular ultrasound (IVUS), and now by newer methods such as optical coherence tomography (OCT), blood tests, and coronary computed tomography angiography (CCTA). We will focus this review on the pathogenesis (including the role of donor-specific antibodies [DSAs]) and diagnosis of CAV.

\section{Epidemiology}

The prevalence of CAV rises over time and appears to increase almost exponentially beyond 5 years, with an approximate $10 \%$ increase in incidence during each 2-year interval after OHT. ${ }^{5}$ Data from recent International Society of Heart and Lung Transplantation (ISHLT) registry estimate the prevalence of angiographically detected CAV at 8\%-10\% at 1 year, $20 \%$ at 3 years, $30 \%$ at 5 years, $45 \%$ at 8 years, and $>50 \%$ at 10 years. ${ }^{2,6,7}$ This contrasts with older estimates of CAV rates as high as $40 \%-50 \%$ by 5 years after OHT, suggesting delayed and/or declining CAV development with modern posttransplant care. ${ }^{8,9}$ In addition, the true prevalence of disease depends on the screening method and definition used for CAV. Intravascular imaging with IVUS or OCT has been shown to be more sensitive than coronary angiography for detecting CAV. At 4-5 years after transplantation, the diagnosis of CAV is made in about $55 \%$ of patients by IVUS or OCT, but only in $33 \%$ by angiography. ${ }^{10}$ On the basis of autopsy studies, as many as $75 \%$ of transplant patients with angiographically normal coronaries had significant intimal hyperplasia, although this was not the cause of death in all patients. ${ }^{11}$

CAV can include both coronary artery disease (CAD) already present in the donor heart and de novo disease that develops in the transplanted heart. It is important to differentiate between the two entities, since there is increasing utilization of expanded donor hearts because of a shortage of available donors, and these may be at higher risk for developing CAV.

\section{Risk factors}

Risk factors for CAV development include those related to the donor, the recipient, and unique transplant-specific immune factors. ${ }^{5,12}$ Major donor-derived risk factors for CAV development include increasing donor age (especially $>40$ years), donor history of hypertension, male donor, and death from head trauma or explosion. ${ }^{6,7,12,13}$ Baseline donor CAD and left ventricular hypertrophy appear to increase the risk of significant CAV (especially early after OHT). $., 14,15$
Recipient risk factors for CAV include higher recipient body mass index, older age, male recipient, prior ischemic heart disease, ventricular assist device support, and recent infection. ${ }^{6,7,12}$ Traditional atherosclerotic risk factors such as hypertension, hyperlipidemia (including triglyceride/highdensity lipoprotein ratio $>3$ ), diabetes, and smoking also increase the risk of CAV. ${ }^{3,5,8,16,17}$

Immune risk factors for CAV include use of Muromonab-CD3 (OKT3) induction, azathioprine (instead of mycophenolate), cyclosporine (instead of tacrolimus), acute rejection, development of DSAs, and cytomegalovirus (CMV) mismatch (seronegative recipient of a seropositive donor organ). ${ }^{6,7,12} \mathrm{CMV}$ infection and increased number of acute rejection episodes during the first year after OHT significantly increase the risk of CAV, and the effects of cellular- and antibody-mediated rejection will be discussed later (Table 1).

\section{Pathogenesis}

Autopsy studies in transplant recipients with established CAV demonstrate that CAV is a combination of intimal fibromuscular hyperplasia, traditional atherosclerosis, and vasculitis,

\section{Table I CAV risk factors}

Donor-derived factors
Older donor age or male donor
Donor CAD
Donor HTN/LVH
Explosive donor death
Recipient-derived factors (non-modifiable)
Older recipient age or male recipient
History of ischemic heart disease
VAD support prior to OHT
Infection prior to OHT
Recipient metabolic factors (modifiable)
HTN
Hyperlipidemia
Insulin resistance
Elevated TG/HDL ratio
Diabetes
Smoking
Higher body mass index
Immunologic factors
Cyclosporine instead of tacrolimus
Azathioprine instead of mycophenolate
Recurrent acute cellular rejection
Antibody-mediated rejection
CMV infection
Donor-recipient HLA mismatches
Circulating anti-HLA antibodies

Abbreviations: CAV, cardiac allograft vasculopathy; CAD, coronary artery disease; HTN, hypertension; LVH, left ventricular hypertrophy; VAD, ventricular assist device; $\mathrm{OHT}$, orthotopic heart transplant; TG, triglyceride; HDL, high-density lipoprotein; CMV, cytomegalovirus; HLA, human leukocyte antigen. 
intimal hyperplasia of the small and large coronary arteries being the most common finding. ${ }^{18}$

Although not completely understood, it is thought that the pathogenesis of CAV involves repeated injuries to the endothelium from a variety of factors such as cellular-mediated rejection, alloimmune factors, ischemiareperfusion injury at time of transplant, CMV infection, immunosuppression medications, systemic inflammation, and traditional atherosclerosis risk factors. ${ }^{3,19-21}$ A schematic and theoretical progression of disease is illustrated below. Damage to the endothelium leads to smooth muscle cell proliferation, infiltration of the intima with inflammatory cells, and collagen deposition. ${ }^{22}$ This process causes the evolution from focal intimal thickening early after transplant to circumferential diffuse thickening and development of atherosclerotic plaques at later stages. ${ }^{23,24}$ The absolute amount of intimal hyperplasia tends to be constant in the entire arterial tree but is more noticeable in the distal vasculature given the smaller lumen size ${ }^{25}$ (Figures 1 and 2).

Atherosclerotic coronary lesions already present in the donor heart at the time of transplantation can rapidly progress after transplantation, leading to focal stenosis that usually affects proximal portions of the major epicardial coronary arteries. ${ }^{21}$ The influence of traditional atherosclerotic risk factors such as older donor age, hypertension, left ventricular hypertrophy, and donor coronary disease emphasizes the overlapping pathophysiology..$^{5-7,21}$ Early CAV, defined as within the first year after heart transplantation, reflects either progression of donor-derived CAD (especially focal, proximal lesions) or an aggressive CAV phenotype that behaves like an inflammatory vasculitis. Although preexisting donor-derived atherosclerosis can become clinically significant, whether all such plaques will progress is not fully understood. One IVUS-based study found that the intimal thickness of donor-derived atherosclerotic lesions did not progress faster than other segments, nor were they associated with higher mortality, arguing against progression of donor-derived lesions as the dominant cause of CAV. ${ }^{14}$ This is in contrast to the early-onset inflammatory vasculitis-type CAV, which does carry a worse prognosis. ${ }^{1}$

Immunosuppression medications and CMV may both directly affect the coronary endothelium. Cyclosporine, which is a frequently used immunosuppression agent, may directly impair coronary microvascular endothelial function when compared with tacrolimus. This, over time, can lead to greater intimal thickening. ${ }^{26} \mathrm{CMV}$ infection can also play a role in CAV development by direct endothelial cell injury and induction of proinflammatory cytokines ${ }^{3,27}$ (Figure 3 ).

\section{Role of rejection}

Solid organ transplantation induces a cellular reaction responsible for acute graft rejection and is a risk factor for developing CAV. Rates of cellular rejection are lower now with advances in induction and immunosuppressive therapies. However, development of CAV is much more complex than simply being due to low levels of chronic cellular rejection. The role of the humoral immune system and

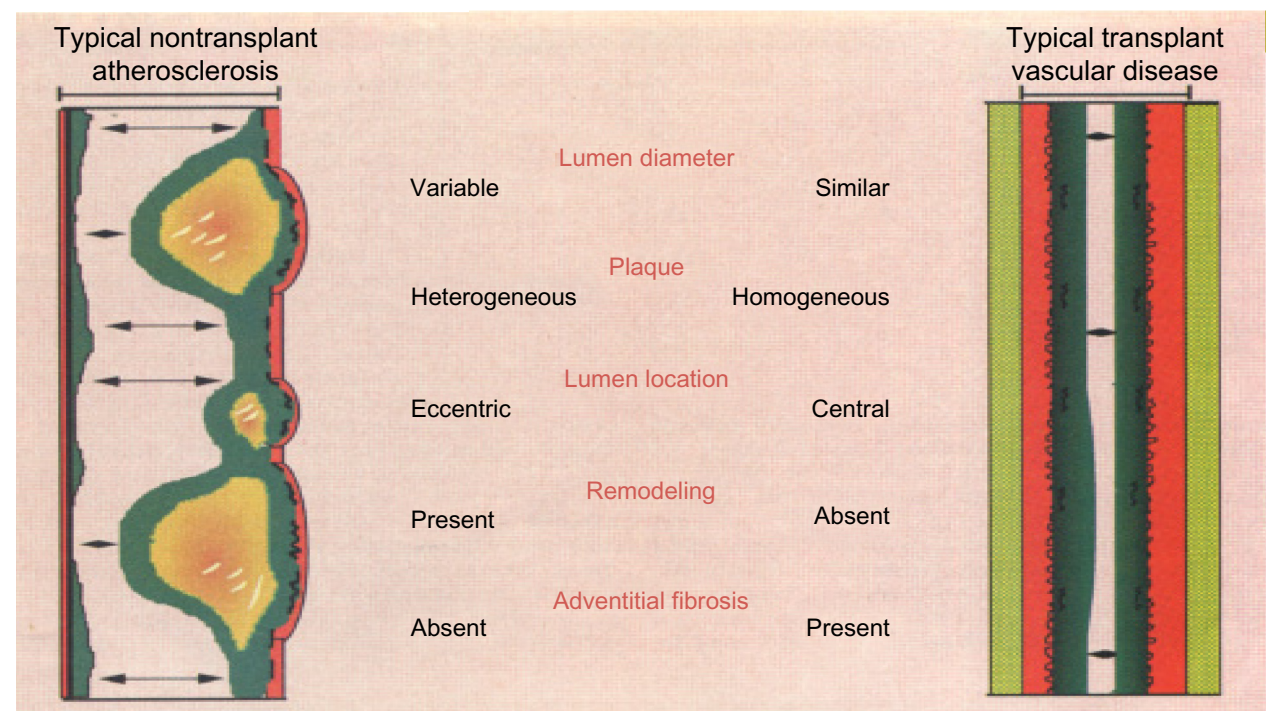

Figure I Differences between classic atherosclerosis and transplant vascular disease or cardiac allograft vasculopathy.

Note: Reprinted from The American Journal of Cardiology, 78(7), Arbustini E, Roberts WC, Morphologic observations in the epicardial coronary arteries and their surroundings late after cardiac transplantation (allograft vascular disease), 814-820, Copyright 1996, with permission from Elsevier. ${ }^{76}$ 


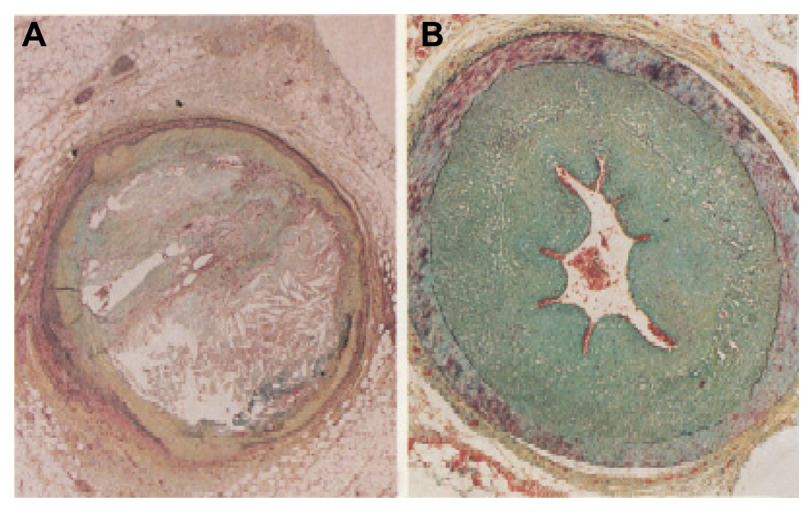

Figure 2 Photomicrographs of typical atherosclerosis (A) and cardiac allograft vasculopathy with neointimal hyperplasia (B).

Note: Reprinted from The American Journal of Cardiology, 78(7), Arbustini E, Roberts WC, Morphologic observations in the epicardial coronary arteries and their surroundings late after cardiac transplantation (allograft vascular disease), 814-820, Copyright 1996, with permission from Elsevier. ${ }^{76}$

how antibody-mediated injury leads to CAV is just starting to be understood and is felt to play a significant role. ${ }^{28,29}$ Host immune response against donor human leukocyte antigen (HLA), and non-HLA and endothelial antigens can lead to damage to the coronary endothelium of the transplanted heart. Donor coronary endothelial injury and endothelial dysfunction represent the earliest step in development of $\mathrm{CAV}$, provoking an alloimmune inflammatory response which leads to an excessive repair process. ${ }^{3,21}$ This excessive repair process causes progressive diffuse luminal narrowing involving the entire coronary tree because of circumferential intimal smooth muscle cell proliferation and extracellular matrix production..$^{20,21}$ Roughly $9 \%$ of patients who undergo transplantation are sensitized to HLA antibodies (panel reactive antigen $>10 \%$ ) at the time of the transplant. Clinical outcomes in these patients are worse because of increased rejection and $\mathrm{CAV}^{2}$ We now know that even patients with a negative cross-match at the time of transplant can develop de novo DSAs to HLA and non-HLA antigens after transplantation, which may play a role in the development of CAV. There is no way to predict who will develop these antibodies if they are not present at the time of transplant, and it is unknown if they trigger the cascade leading to CAV.

With newer Luminex technology (Luminex, Austin, TX, USA), determination of the actual DSAs that are present at the time of transplant is possible, and studies suggest that $33 \%$ of patients will develop DSAs after OHT. ${ }^{30-33}$ Luminex works by attaching each specific HLA and non-HLA antigens of interest to unique fluorescent microscopic beads, and the unique fluorescent emission for each microsphere allows for determination of the type of antibodies are present. These de novo antibodies may not be present until several months after transplant, perhaps because of greater early immunosuppression and the lag time necessary for the development of antibodies after antigen exposure. The mere presence of DSAs is not necessarily indicative that these antibodies are cytotoxic and will damage the endothelium. In order to damage the endothelium of the transplanted heart, these

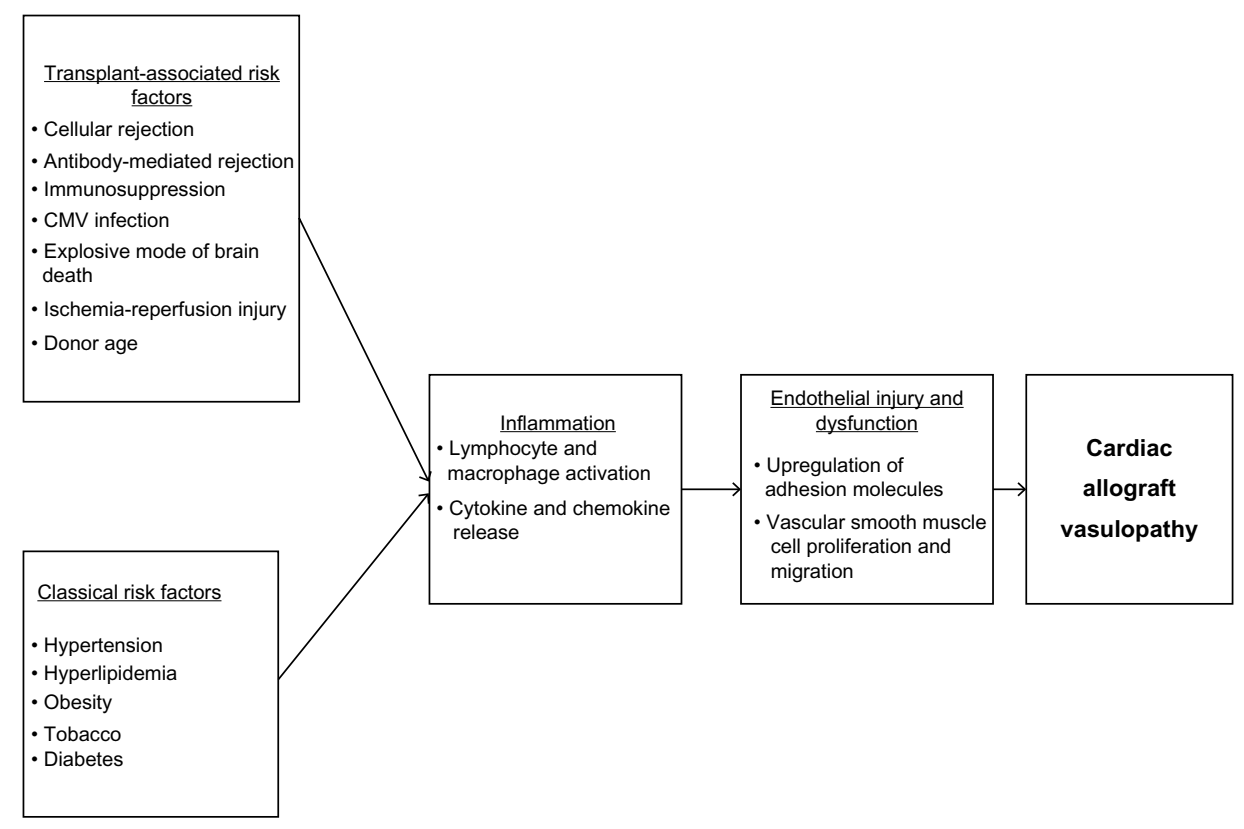

Figure 3 Risk factors and pathogenesis of cardiac allograft vasculopathy.

Note: Reproduced from Cardiac allograft vasculopathy: current knowledge and future direction, Colvin-Adams M, Agnihotri A, Clin Transplant, 20 II;25(2):I75-I84. Copyright @ 201 I John Wiley and Sons A/S. Available from: http://onlinelibrary.wiley.com/doi/l 0.1 III/j.1399-0012.2010.01307.x/abstract. ${ }^{3}$

Abbreviation: CMV, cytomegalovirus. 
antibodies must activate the complement system. There is now a novel Luminex-based assay that detects $\mathrm{C} 1 \mathrm{q}$ binding to HLA and non-HLA antibodies and thus detects the antibodies that trigger complement activation. ${ }^{34-37}$ This $\mathrm{C} 1 \mathrm{q}$ binding DSA by Luminex was recently shown to predict renal graft survival, but the correlation of C1q versus non-C1q binding DSA with intimal thickening and CAV is still under investigation. ${ }^{37}$

CAV and rejection can occur even in patients without measurable HLA antibodies. Therefore, other antigens that can trigger an immune response injury have been sought. Other antibodies against antigens such as major histocompatibility-complex class I chain-related antigens A (MICA) have recently been associated in cohorts of patients with documented CAV. MICA antigens are expressed on endothelial cells, and DSAs to MICA can trigger complement-dependent injury as well. In a retrospective study by Nath et al, ${ }^{38} 67 \%$ of patients with angiographic evidence of CAV had DSAs against HLA or MICA, whereas $33 \%$ of patients without CAV had these DSAs. In a similar manner, Zhang et al ${ }^{39}$ concluded that patients with DSAs against HLA or MICA had a 77\% survival free of CAV at 2 years, defined by angiographic stenosis of $>50 \%$, compared with $96 \%$ in those without these DSAs. However, neither study correlated DSA with complement activation, endothelial function, or intimal thickening. Although these studies suggest an association, they do not answer the question of causality.

The role of immune responses to other vascular antigens such as vimentin, myosin, and angiotensin II type 1 receptor has been linked to antibody-mediated rejection, but their role in CAV is even less clear. ${ }^{40}$ Measuring for antibodies against these antigens is more difficult given the lack of standardized reagents, and in the future, it may be another area of research.

Prospective data on whether DSAs that activate complement are associated with endothelial dysfunction, and their relationship with developing intimal thickening is not well established, and warrants further studies.

\section{Diagnosis}

Patients with significant CAV are often asymptomatic and rarely present with angina because of cardiac sensory denervation. This makes the diagnosis of CAV difficult and routine screening crucial. Manifestations of CAV include dyspnea, heart failure, syncope, palpitations, graft dysfunction, and even ventricular arrhythmias resulting in sudden cardiac death. ${ }^{3}$ Current guidelines recommend the use of routine screening for CAV in asymptomatic OHT patients, as well as diagnostic testing for CAV in patients with worsening cardiovascular symptoms, arrhythmias,

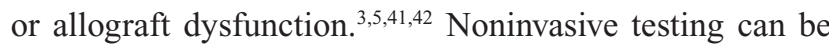
performed by stress myocardial perfusion imaging or stress echocardiography. Angiography is the most common method of screening; however, its limitation is that it visualizes only luminal diameter and cannot measure intimal hyperplasia. IVUS and virtual histology-IVUS have advanced our knowledge and detection of CAV by allowing for measurement of intima-media thickness and assessment of plaque, but are limited by their resolution of 100-300 $\mu \mathrm{m}$. Therefore, other ways to diagnose $\mathrm{CAV}$, such as endothelial function testing, OCT imaging, and even computed tomography (CT) angiography, are under investigation and now being clinically utilized.

There is hope that CAV may be detected through serum blood tests such as by biomarkers or DNA and microRNA testing. Elevated levels of cardiac troponin, B-type natriuretic peptide (BNP), and C-reactive protein (CRP) after OHT (particularly if persistent or worsening) are all associated with development of CAV as well as graft failure with or without adverse cardiac events. ${ }^{1,3}$ Beyond these traditional markers, none are sensitive enough to become part of clinical practice just yet.

\section{Noninvasive testing}

The role of noninvasive screening tests for CAV diagnosis remains controversial, and each test has important limitations. ${ }^{1,3,43}$ Traditional stress testing is designed to detect flow-limiting coronary disease and is likely not effective for early detection of CAV because ischemia does not occur until there is significant luminal narrowing of at least $70 \%$ or severe distal disease. The ability to avoid the need for invasive testing, nephrotoxic contrast agents, and/or ionizing radiation makes their selective use appealing for patients with kidney disease or those who remain free from significant CAV during late follow-up. ${ }^{3,41,43}$ Stress testing by either nuclear perfusion imaging or dobutamine stress echocardiography has good sensitivity (up to $85 \%-90 \%$ ) and negative predictive value $(>95 \%)$ for angiographically significant CAV lesions, but is far less sensitive for milder preclinical CAV. These tests provide prognostic information, since a positive test result usually signifies advanced disease. , $3,43,44^{-}$

Other parameters on stress testing have been studied as possible markers for CAV. Systolic dysfunction and wall motion abnormalities on resting echocardiography or reduced mitral annular systolic tissue Doppler velocities (s') $\leq 10 \mathrm{~cm} / \mathrm{s}$ 
signify significant CAV, but their use is not part of routine clinical care. ${ }^{45}$

CCTA has a sensitivity of up to $89 \%$ and an excellent negative predictive value of $97 \%$ for $\mathrm{CAV}^{46}$ In a recent meta-analysis, the newest-generation CCTA ( $\geq \geq 16$ slices) was compared to coronary angiography and IVUS and the analysis showed that CCTA was accurate in excluding CAV in the majority of OHT patients, especially when compared to standard coronary angiography. When compared to IVUS, the sensitivity and negative predictive value of CCTA was lower ( $81 \%$ and $50 \%$, respectively), likely because CT is designed to assess the lumen size and not intimal hyperplasia. ${ }^{46}$ CCTA is less invasive than coronary angiography and IVUS, less costly, and less resource intensive. ${ }^{47}$ However, the limitations of CCTA include degradation of image quality due to elevated resting heart rate (typical for OHT recipients), need for nephrotoxic contrast agents, and high doses of ionizing radiation. ${ }^{1,43,48}$ Despite these, the use of CCTA may be reasonable in selected stable OHT patients with preserved renal function as a screening tool to exclude significant obstructive CAV. ${ }^{47}$

At our institution, we prefer dobutamine stress echocardiography as an alternative to coronary angiography for patients with significant kidney disease (creatinine clearance $[\mathrm{CrCl}]<30 \mathrm{~mL} / \mathrm{min}$ or creatinine $>2.5 \mathrm{mg} / \mathrm{dL}$ ), advanced age, comorbidities that may limit their survival, and those without CAV late after transplant.

\section{Invasive testing}

Coronary angiography is the standard diagnostic test for CAV and is recommended if CAV is suspected. The sensitivity of coronary angiography alone for CAV is suboptimal because of the diffuse nature of the disease and lack of a normal reference vessel for grading stenosis in many cases. $3,5,20$ Despite the lack of sensitivity for early CAV lesions, angiography provides important prognostic information and reliably excludes obstructive CAV. ${ }^{3}$ The International Society of Heart Lung Transplantation (ISHLT) has developed a nomenclature to help standardize the way CAV is reported and compared which has become the preferred classification system $^{1}$ (Figure 4).

The ISHLT CAV grading system incorporates CAV extent and severity as well as allograft function to encompass the entire spectrum of CAV burden. Angiographic grading systems such as that described by Gao et $\mathrm{al}^{49}$ categorize the variable angiographic appearance of CAV and are more descriptive in nature, but might also help to identify focal CAV lesions that are more amenable to revascularization (Figure 5).

Intravascular imaging can be performed by two modalities, and each has certain advantages. IVUS has become the standard for the diagnosis of preclinical CAV in view of its ability to measure intima and media thickness. The IVUS-derived parameters of coronary maximal media-intima thickness (MIT), maximal media-intimal area (MIA), mean intimal thickness, and atheroma volume offer important prognostic information. ${ }^{1,3,5,20,50,51}$ Rapidly progressive CAV, defined by an increase in MIT $\geq 0.5 \mathrm{~mm}$ and/or an increase in MIA $\geq 3.5 \mathrm{~mm}^{2}$ between baseline and 1 year, is associated with increased rates of death, graft loss, and nonfatal adverse cardiac events. ${ }^{41,50,52}$ In the absence of CAV at 1 year, coronary angiography can proceed every 1-2 years until year 3-5, after which patients without established CAV may undergo less-frequent invasive testing. ${ }^{41}$

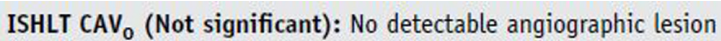

ISHLT CAV 1 (Mild): Angiographic left main (LM) $<50 \%$, or primary vessel with maximum lesion of $<70 \%$, or any branch stenosis $<70 \%$ (including diffuse narrowing) without allograft dysfunction

ISHLT CAV ${ }_{2}$ (Moderate): Angiographic LM $<50 \%$; a single primary vessel $\geq 70 \%$, or isolated branch stenosis $\geq 70 \%$ in branches of 2 systems, without allograft dysfunction

ISHLT $\mathrm{CAV}_{3}$ (Severe): Angiographic LM $\geq 50 \%$, or two or more primary vessels $\geq 70 \%$ stenosis, or isolated branch stenosis $\geq 70 \%$ in all 3 systems; or ISHLT CAV1 or CAV2 with allograft dysfunction (defined as LVEF $\leq 45 \%$ usually in the presence of regional wall motion abnormalities) or evidence of significant restrictive physiology (which is common but not specific; see text for definitions)

\section{Definitions}

a). A "Primary Vessel" denotes the proximal and Middle 33\% of the left anterior descending artery, the left circumflex, the ramus and the dominant or co-dominant right coronary artery with the posterior descending and posterolateral branches.

b). A "Secondary Branch Vessel" includes the distal $33 \%$ of the primary vessels or any segment within a large septal perforator, diagonals and obtuse marginal branches or any portion of a non-dominant right coronary artery.

c). Restrictive cardiac allograft physiology is defined as symptomatic heart failure with echocardiographic $E$ to $A$ velocity ratio $>2$ ( $>1.5$ in children), shortened isovolumetric relaxation time ( $<60 \mathrm{msec})$, shortened deceleration time ( $<150 \mathrm{msec})$, or restrictive hemodynamic values (Right Atrial Pressure $>12 \mathrm{mmHg}$, Pulmonary Capillary Wedge Pressure $>25 \mathrm{mmHg}$, Cardiac Index $<2(/ \mathrm{min} / \mathrm{m} 2)$

Figure 4 Recommended nomenclature for cardiac allograft vasculopathy.

Note: Reprinted from The Journal of Heart and Lung Transplantation, 29(7), Mehra MR, Crespo-Leiro MG, Dipchand A, et al. International Society for Heart and Lung Transplantation working formulation of a standardized nomenclature for cardiac allograft vasculopathy-2010, 7I7-727, Copyright 20I0, with permission from Elsevier.'

Abbreviations: ISHLT, International Society of Heart and Lung Transplantation; CAV, coronary allograft vasculopathy; LVEF, left ventricular ejection fraction. 


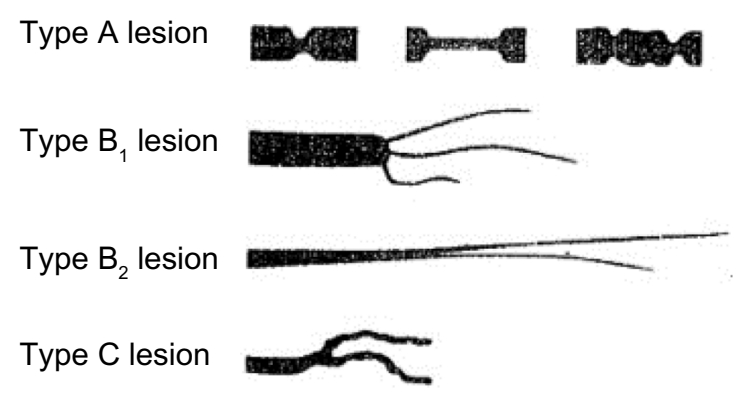

Figure 5 Anatomic abnormalities in transplant vascular coronary artery disease. Notes: Type A lesion: discrete, tubular, or multiple stenosis. Type $B_{1}$ lesion: abrupt onset with diffuse distal concentric narrowing and obliterated vessels. Type $B_{2}$ lesion: gradual, concentric tapering with distal portion having some residual lumen. Type C lesion: narrowed, irregular distal branches with terminations that are often nontapered and squared off, ending abruptly. Reprinted from JAm Coll Cardiol. 1988; 12 , Gao SZ, Alderman EL, Schroeder JS, Silverman JF, Hunt SA. Accelerated coronary vascular disease in the heart transplant patient: coronary arteriographic findings. 334-340. Copyright $@ 1988$ with permission from Elsevier. ${ }^{49}$

Recent studies have evaluated the use of OCT in detecting CAV because of its excellent spatial recognition of 10-20 $\mu \mathrm{m}$, which is 10 -fold greater than that with IVUS.$^{53}$ The high resolution and the ability to measure the intima and media layers individually make this theoretically the ideal screening tool. The recent OCT CAV study included 15 patients without angiographic evidence of CAV and used an intima/ media (I/M) ratio of $>1$ as a cutoff for diagnosing CAV. In this population, $53 \%$ had evidence of CAV despite normal angiography. ${ }^{25}$ Additionally, towing to the spatial resolution, quantification of atherosclerosis, including lipid-rich and calcified plaques, can be performed with OCT. Recently, OCT was discovered to have the ability to distinguish newly developed CAV from donor-transmitted atherosclerosis, potentially allowing for earlier diagnosis of true CAV and providing a metric to gauge effectiveness of CAV treatment ${ }^{54}$ (Figures 6 and 7).

Many comparisons have been made between these modalities. IVUS technology has been available longer and more readily available at catheterization laboratories worldwide. With a resolution of $200 \mu \mathrm{m}$, IVUS is better suited to detect severe disease defined as greater than $500 \mu \mathrm{m}$. IVUS also has more historical data and certainly provides important prognostic information based on prior longitudinal studies. ${ }^{50,52}$ OCT, on the other hand, has a 10-fold higher resolution and can measure the intima layer separately from the medial layer, detect subtle changes in intimal hyperplasia, and quantify atherosclerotic plaques better. Unlike IVUS, OCT does require additional contrast administration during coronary angiography, which can be problematic in patients with kidney disease. Additional OCT-based studies are needed to determine the clinical significance of these findings and how they correlate with outcomes such as graft survival.

Other invasive measurements of coronary microvascular function have been explored to allow for earlier diagnosis of CAV, including thrombolysis in myocardial infarction (TIMI) frame count and coronary flow reserve. ${ }^{1,3}$ TIMI frame counting is performed by measuring the number of frames until contrast dye reaches the end of a vessel during coronary angiography and is increased by microvascular disease. Coronary flow reserve utilizes a Doppler wire in the coronary artery to measure distal blood flow based on the concept that flow is reduced with microvascular disease during hyperemia. Several findings on endomyocardial biopsy, including stenotic microvasculopathy, endothelialitis, and perivascular fibrosis, have been identified as insensitive predictors of CAV. ${ }^{1,20}$
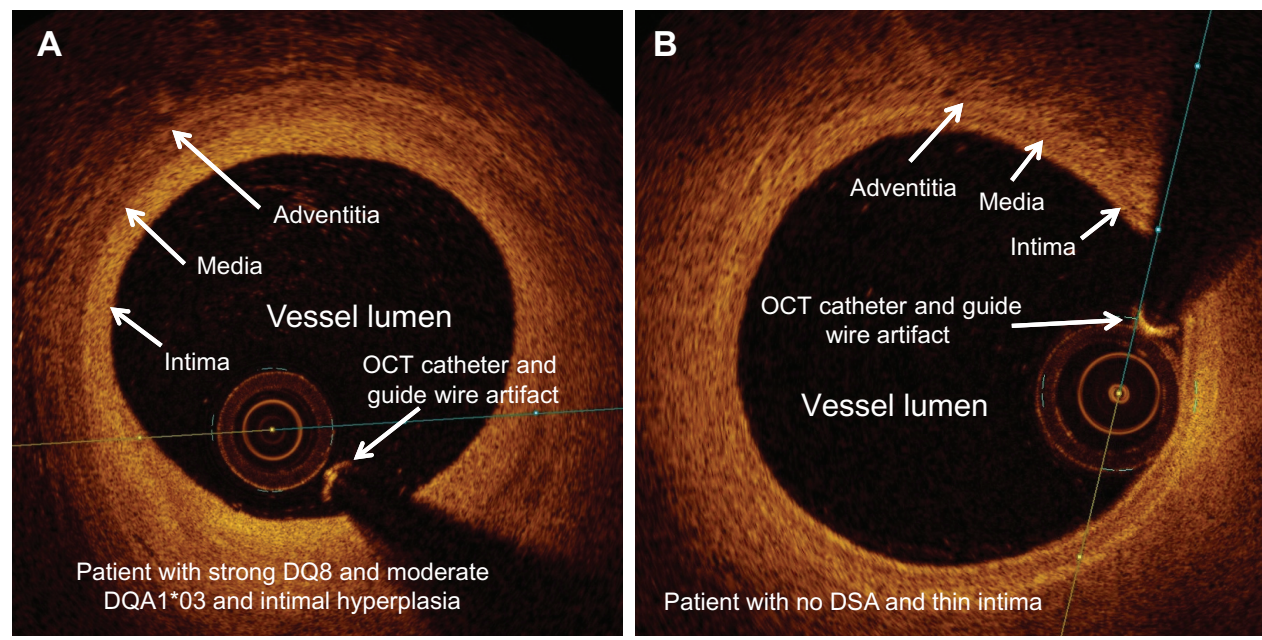

Figure 6 OCT imaging of coronary artery of a heart transplant patient with significant intimal hyperplasia and known antibody-mediated rejection (A) and a patient with normal-appearing intima without antibodies (B).

Abbreviations: OCT, optimal coherence tomography; DSA, donor-specific antibodies. 

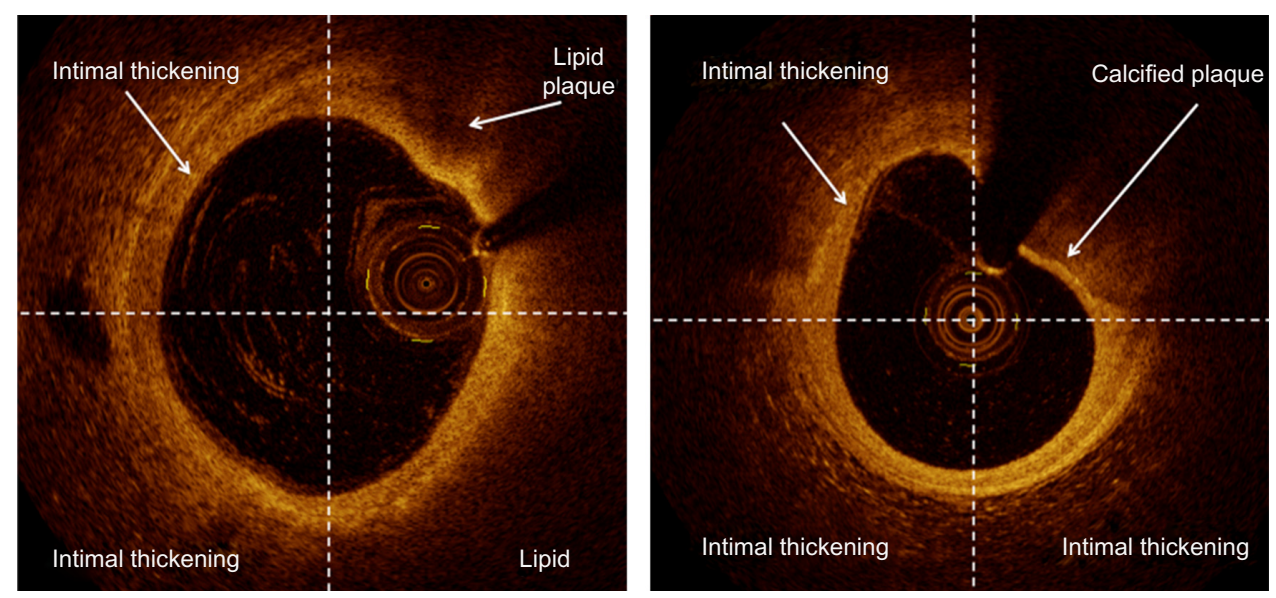

Figure 7 Optimal coherence tomography (OCT) imaging of coronary artery in a heart transplant patient showing the ability of OCT to characterize intimal hyperplasia and various types of plaques.

\section{Prevention and treatment}

Given the risk associated with CAV and limited treatment options, prevention of CAV is paramount for optimal OHT outcomes. ${ }^{55}$ Many of the same treatment options for traditional atherosclerosis have been applied to CAV. Statins are the cornerstone of primary CAV prevention by lowering lipid levels and attenuating the adverse effects of hyperlipidemia on CAV development. ${ }^{3,17,55}$ In addition, the pleiotropic effects of statins may inhibit alloimmune responses, including CMV replication. Statins are recommended for all OHT recipients regardless of cholesterol levels on the basis of randomized trial data showing reduced rates of CAV, all-cause mortality, and hemodynamically significant rejection with statin treatment. ${ }^{3,17,41,55-60}$ Hypertension control is important for CAV prevention, and both angiotensin converting enzyme (ACE) inhibitors and calcium-channel blockers appear to reduce CAV development and/or progression. . $^{3,16,61} \mathrm{As}$ with traditional CAD, prevention and control of diabetes after OHT likely retards CAV development. Prevention of CMV infection is critical to reducing the risk of developing CAV. Universal prophylaxis has been shown to be more effective than preemptive therapy for reducing CAV risk (Table 2). ${ }^{3,27,62,63}$

The choice of maintenance immunosuppression regimen likely influences CAV risk after transplantation either by preventing rejection or from direct effects of the drugs. The combination of tacrolimus and mycophenolate has become the most popular combination of routine immunosupression due to its efficacy at preventing cellular rejection and the concept that preventing acute rejeciton will lower the risk of CAV. ${ }^{2,5-7,55}$ The proliferation signal inhibitor (PSI) drugs sirolimus and everolimus are potent inhibitors of intimal proliferation, and for this reason, they have become the drugs of choice for drug-eluting coronary stents to prevent in-stent restenosis, a process analogous to CAV progression. ${ }^{64}$

Regimens utilizing a PSI are appealing because the use is associated with reduced rates of CAV and improved renal function. ${ }^{55,65,66}$ Current guidelines do not recommend the use of PSI for immunosuppression immediately after OHT in view of the lack of mortality benefit, fear of higher cellular rejection rates, and other adverse effects, including poor wound healing. ${ }^{41}$ Current guidelines suggest adding a PSI to ongoing calcineurin inhibitor therapy in place of azathioprine or mycophenolate for patients with established CAV. ${ }^{41}$ There are ongoing studies to look at the ideal combination and timing of immunosuppression agents, including early use of PSI once healing from the transplant surgery is complete. Regimens continue to vary between centers, and a consensus has not yet been reached.

Other nontraditional agents have been studied as well. The combination of antioxidant vitamins $\mathrm{C}$ and $\mathrm{E}$ reduced

\section{Table 2 CAV prevention}

Statins
Antihypertensives
ACE inhibitors
Calcium channel blockers
Immunosuppression
Tacrolimus (instead of cyclosporine)
Mycophenolate (instead of azathrioprine)
Sirolimus/everolimus (in higher risk patients)
Cytomegalovirus prophylaxis
Glycemic control

Abbreviations: $C A V$, cardiac allograft vasculopathy; $A C E$, angiotensin converting enzyme. 
CAV progression in a small trial and can be useful when modification of the immunosuppression regimen is inappropriate or unsuccessful. ${ }^{20,67}$ Small animal studies using aortic allografts have suggested that platelet inhibition with clopidogrel might antagonize the development of allograft vasculopathy in a manner synergistic with everolimus but not cyclosporine, although human data are still lacking. ${ }^{68,69}$ The role for traditional antianginal therapy with $\beta$-blockers and nitrates is limited to symptom management. Patients with advanced CAV leading to systolic dysfunction who are candidates for retransplantation may benefit from implantable cardiac defibrillator placement, since death is often caused by ventricular arrhythmias. ${ }^{42,55,70,71}$

Given the diffuse nature of $\mathrm{CAV}$, revascularization is often not feasible, although both surgical and percutaneous revascularization can be successful for discrete proximal CAV lesions. ${ }^{5,44,55}$ Percutaneous coronary intervention (PCI) with stenting is preferred for patients with focal lesions amenable to this procedure. Restenosis rates are $18 \%$ with drug-eluting stents and up to $43 \%$ with bare-metal stents. These rates are higher than in the general population, and therefore, drug-eluting stent use is preferable as long as patients can tolerate longer-duration dual antiplatelet therapy. ${ }^{5,41,55,64,72}$ Long-term survival in patients with CAV amenable to PCI is greater than that in those with severe CAV not amenable to $\mathrm{PCI} .{ }^{73}$ Fewer OHT recipients with CAV are candidates for coronary artery bypass grafting (CABG) than for PCI, but the highly-selective $\mathrm{CAV}$ patients undergoing $\mathrm{CABG}$ may have favorable outcomes. ${ }^{5,44,55} \mathrm{CAV}$ is the most common indication for retransplantation, which remains the definitive therapy when CAV is not amenable to revascularizations (Table 3)..$^{2,5-7,41,55,74,75}$

\section{Table 3 CAV treatment options}

Statins
Goal LDL $<70 \mathrm{mg} / \mathrm{dL}$
Antihypertensives
ACE-inhibitors
Calcium channel blockers
Beta-blockers/nitrates for angina
Immunosuppression
Sirolimus/everolimus in place of mycophenolate/tacrolimus
Clopidogrel
Antioxidants (vitamin C and E)
Revascularization
PCI with drug-eluting stents for amenable lesions
CABG in highly selected patients
Re-transplantation for end-stage CAV or ischemic cardiomyopathy

Abbreviations: CAV, cardiac allograft vasculopathy; LDL, low-density lipoprotein; $\mathrm{ACE}$, angiotensin converting enzyme; $\mathrm{PCl}$, percutaneous coronary intervention; CABG, coronary artery bypass grafting.

\section{Conclusion}

CAV is common after OHT and associated with significant morbidity and long-term mortality. Early diagnosis can be difficult given the lack of symptoms due to cardiac sensory denervation and the diffuse nature of CAV. Given the poor prognosis once clinically apparent CAV occurs, a variety of screening methods have been investigated. Coronary angiography has recently been supplanted by intravascular imaging as the gold standard for diagnosing CAV, and further studies are necessary to compare IVUS and OCT as the imaging tool of choice. Recent advances in medical therapy aimed at primary prevention of CAV utilizing statins, CMV prophylaxis, and lower cellular rejection rates have resulted in a decrease in prevalence of CAV and improvement in prognosis. Studies are also ongoing for treatment options once CAV develops, including PSI-based immunosuppression. Although great strides have been made in recent years, randomized trials will need to be performed to optimize the immunosuppression regimens of OHT patients, especially in the first year after transplant, to reduce the prevalence and incidence of CAV.

\section{Disclosure}

The authors report no conflicts of interest in this work.

\section{References}

1. Mehra MR, Crespo-Leiro MG, Dipchand A, et al. International Society for Heart and Lung Transplantation working formulation of a standardized nomenclature for cardiac allograft vasculopathy-2010. J Heart Lung Transplant. 2010;29(7):717-727.

2. Stehlik J, Edwards LB, Kucheryavaya AY, et al. The Registry of the International Society for Heart and Lung Transplantation: twenty-seventh official adult heart transplant report-2010. J Heart Lung Transplant. 2010;29:1089-1103.

3. Colvin-Adams M, Agnihotri A. Cardiac allograft vasculopathy: current knowledge and future direction. Clin Transplant. 2011;25(2): $175-184$.

4. Prada-Delgado O, Estevez-Loureiro R, Paniagua-Martin MJ, Lopez-Sainz A, Crespo-Leiro MG. Prevalence and prognostic value of cardiac allograft vasculopathy 1 year after heart transplantation according to the ISHLT recommended nomenclature. J Heart Lung Transplant. 2012;31:332-333.

5. Zimmer RJ, Lee MS. Transplant coronary artery disease. JACC Cardiovasc Interv. 2010;3:367-377.

6. Stehlik J, Edwards LB, Kucheryavaya AY, et al. The Registry of the International Society for Heart and Lung Transplantation: 29th official adult heart transplant report-2012. J Heart Lung Transplant. 2012;31:1052-1064.

7. Stehlik J, Edwards LB, Kucheryavaya AY, et al. The Registry of the International Society for Heart and Lung Transplantation: twenty-eighth adult heart transplant report-2011. J Heart Lung Transplant. 2011;30:1078-1094.

8. Hunt SA, Haddad F. The changing face of heart transplantation. $J A m$ Coll Cardiol. 2008;52:587-598.

9. Lindenfeld J, Page RL II, Zolty R, et al. Drug therapy in the heart transplant recipient: III, common medical problems. Circulation. 2005;111:113-117. 
10. Torres HJ, Merello L, Ramos SA, et al. Prevalence of cardiac allograft vasculopathy assessed with coronary angiography versus coronary vascular ultrasound and virtual histology. Transplant Proc. 2011;43:2318-2321.

11. Johnson TH, McDonald K, Nakhleh R, et al. Allograft vasculopathy and death in a cardiac transplant patient with angiographically normal coronary arteries. Cathet Cardiovasc Diagn. 1991;24:37-40.

12. Braga JR, Santos IS, McDonald M, Shah PS, Ross HJ. Factors associated with the development of cardiac allograft vasculopathy - a systematic review of observational studies. Clin Transplant. 2012;26:E111-E124.

13. Lietz K, John R, Mancini DM, Edwards NM. Outcomes in cardiac transplant recipients using allografts from older donors versus mortality on the transplant waiting list: implications for donor selection criteria. J Am Coll Cardiol. 2004;43:1553-1561.

14. Li H, Tanaka K, Anzai H, et al. Influence of pre-existing donor atherosclerosis on the development of cardiac allograft vasculopathy and outcomes in heart transplant recipients. J Am Coll Cardiol. 2006;47:2470-2476.

15. Goland S, Czer LS, Kass RM, et al. Use of cardiac allografts with mild and moderate left ventricular hypertrophy can be safely used in heart transplantation to expand the donor pool. $J$ Am Coll Cardiol. 2008;51:1214-1220.

16. Rockx MA, Haddad H. Use of calcium channel blockers and angiotensin-converting enzyme inhibitors after cardiac transplantation. Curr Opin Cardiol. 2007;22:128-132.

17. Bilchick KC, Henrikson CA, Skojec D, Kasper EK, Blumenthal RS. Treatment of hyperlipidemia in cardiac transplant recipients. Am Heart J. 2004;148:200-210.

18. Lu WH, Palatnik K, Fishbein GA, et al. Diverse morphologic manifestations of cardiac allograft vasculopathy: a pathologic study of 64 allograft hearts. J Heart Lung Transplant. 2011;30:1044-1050.

19. Arora S, Gunther A, Wennerblom B, et al. Systemic markers of inflammation are associated with cardiac allograft vasculopathy and an increased intimal inflammatory component. Am J Transplant. 2010;10:1428-1436.

20. Schmauss D, Weis M. Cardiac allograft vasculopathy: recent developments. Circulation. 2008;117:2131-2141.

21. Rahmani M, Cruz RP, Granville DJ, McManus BM. Allograft vasculopathy versus atherosclerosis. Circ Res. 2006;99:801-815.

22. Van Loosdregt J, van Oosterhout MF, Bruggink AH, et al. The chemokine and chemokine receptor profile of infiltrating cells in the wall of arteries with cardiac allograft vasculopathy is indicative of a memory T-helper 1 response. Circulation. 2006;114:1599-1607.

23. Starnes VA, Stinson EB, Oyer PE, et al. Cardiac transplantation in children and adolescents. Circulation. 1987;76:V43-V47.

24. Tuzcu EM, De Franco AC, Goormastic M, et al. Dichotomous pattern of coronary atherosclerosis 1 to 9 years after transplantation: insights from systematic intravascular ultrasound imaging. $\mathrm{J} \mathrm{Am} \mathrm{Coll} \mathrm{Cardiol.}$ 1996;27:839-846.

25. Khandhar SJ, Yamamoto H, Teuteberg JJ, et al. Optical coherence tomography for characterization of cardiac allograft vasculopathy after heart transplantation (OCTCAV study). J Heart Lung Transplant. 2013;32:596-602.

26. Petrakopoulou P, Anthopoulou L, Muscholl M, et al. Coronary endothelial vasomotor function and vascular remodeling in heart transplant recipients randomized for tacrolimus or cyclosporine immunosuppression. J Am Coll Cardiol. 2006;47:1622-1629.

27. Snydman DR, Limaye AP, Potena L, Zamora MR. Update and review: state-of-the-art management of cytomegalovirus infection and disease following thoracic organ transplantation. Transplant Proc. 2011;43:S1-S17.

28. Michaels PJ, Espejo ML, Kobashigawa J, et al. Humoral rejection in cardiac transplantation: risk factors, hemodynamic consequences and relationship to transplant coronary artery disease. J Heart Lung Transplant. 2003;22:58-69.

29. Caforio AL, Tona F, Fortina AB, et al. Immune and nonimmune predictors of cardiac allograft vasculopathy onset and severity: multivariate risk factor analysis and role of immunosuppression. Am J Transplant. 2004;4:962-970.
30. Smith JD, Banner NR, Hamour IM, et al. De novo donor HLA-specific antibodies after heart transplantation are an independent predictor of poor patient survival. Am J Transplant. 2011;11:312-319.

31. Stastny P, Lavingia B, Fixler DE, Yancy CW, Ring WS. Antibodies against donor human leukocyte antigens and the outcome of cardiac allografts in adults and children. Transplantation. 2007;84:738-745.

32. Kaczmarek I, Deutsch MA, Kauke T, et al. Donor-specific HLA alloantibodies: long-term impact on cardiac allograft vasculopathy and mortality after heart transplant. Exp Clin Transplant. 2008;6:229-235.

33. Tambur AR, Pamboukian SV, Costanzo MR, et al. The presence of HLA-directed antibodies after heart transplantation is associated with poor allograft outcome. Transplantation. 2005;80:1019-1025.

34. Chin C, Chen G, Sequeria F, et al. Clinical usefulness of a novel C1q assay to detect immunoglobulin $\mathrm{G}$ antibodies capable of fixing complement in sensitized pediatric heart transplant patients. J Heart Lung Transplant. 2011;30:158-163.

35. Sutherland SM, Chen G, Sequeira FA, Lou CD, Alexander SR, Tyan DB. Complement-fixing donor-specific antibodies identified by a novel C1q assay are associated with allograft loss. Pediatr Transplant. 2012;16:12-17.

36. Chen G, Sequeira F, Tyan DB. Novel C1q assay reveals a clinically relevant subset of human leukocyte antigen antibodies independent of immunoglobulin G strength on single antigen beads. Hum Immunol. 2011;72:849-858.

37. Yabu JM, Higgins JP, Chen G, Sequeira F, Busque S, Tyan DB. C1q-fixing human leukocyte antigen antibodies are specific for predicting transplant glomerulopathy and late graft failure after kidney transplantation. Transplantation. 2011;91:342-347.

38. Nath DS, Angaswamy N, Basha HI, et al. Donor-specific antibodies to human leukocyte antigens are associated with and precede antibodies to major histocompatibility complex class I-related chain $\mathrm{A}$ in antibody-mediated rejection and cardiac allograft vasculopathy after human cardiac transplantation. Hum Immunol. 2010;71:1191-1196.

39. Zhang Q, Cecka JM, Gjertson DW, et al. HLA and MICA: targets of antibody-mediated rejection in heart transplantation. Transplantation. 2011;91:1153-1158.

40. Nath DS, Ilias Basha H, Tiriveedhi V, et al. Characterization of immune responses to cardiac self-antigens myosin and vimentin in human cardiac allograft recipients with antibody-mediated rejection and cardiac allograft vasculopathy. $J$ Heart Lung Transplant. 2010;29:1277-1285.

41. Costanzo MR, Naftel DC, Pritzker MR, et al. Heart transplant coronary artery disease detected by coronary angiography: a multiinstitutional study of preoperative donor and recipient risk factors. Cardiac Transplant Research Database. J Heart Lung Transplant. 1998;17:744-753.

42. Stecker EC, Strelich KR, Chugh SS, Crispell K, McAnulty JH. Arrhythmias after orthotopic heart transplantation. J Card Fail. 2005;11:464-472.

43. Miller CA, Chowdhary S, Ray SG, et al. Role of noninvasive imaging in the diagnosis of cardiac allograft vasculopathy. Circ Cardiovasc Imaging. 2011;4:583-593.

44. Prada-Delgado O, Estevez-Loureiro R, Lopez-Sainz A, et al. Percutaneous coronary interventions and bypass surgery in patients with cardiac allograft vasculopathy: a single-center experience. Transplant Proc. 2012;44:2657-2659.

45. Mondillo S, Maccherini M, Galderisi M. Usefulness and limitations of transthoracic echocardiography in heart transplantation recipients. Cardiovasc Ultrasound. 2008;6:2-14.

46. Wever-Pinzon O, Romero J, Kelesidis I, et al. Coronary computed tomography angiography for the detection of cardiac allograft vasculopathy: a meta-analysis of prospective trials. $\mathrm{J} \mathrm{Am} \mathrm{Coll} \mathrm{Cardiol}$. 2014;63:1992-2004.

47. Kobashigawa J. Coronary computed tomography angiography: is it time to replace the conventional coronary angiogram in heart transplant patients? J Am Coll Cardiol. 2014;63:2005-2006. 
48. Khan R, Jang IK. Evaluation of coronary allograft vasculopathy using multi-detector row computed tomography: a systematic review. Eur J Cardiothorac Surg. 2012;41:415-422.

49. Gao SZ, Alderman EL, Schroeder JS, Silverman JF, Hunt SA. Accelerated coronary vascular disease in the heart transplant patient: coronary arteriographic findings. J Am Coll Cardiol. 1988;12:334-340.

50. Kobashigawa JA, Tobis JM, Starling RC, et al. Multicenter intravascular ultrasound validation study among heart transplant recipients: outcomes after five years. J Am Coll Cardiol. 2005;45:1532-1537.

51. Logani S, Saltzman HE, Kurnik P, Eisen HJ, Ledley GS. Clinical utility of intravascular ultrasound in the assessment of coronary allograft vasculopathy: a review. J Interv Cardiol. 2011;24:9-14.

52. Tuzcu EM, Kapadia SR, Sachar R, et al. Intravascular ultrasound evidence of angiographically silent progression in coronary atherosclerosis predicts long-term morbidity and mortality after cardiac transplantation. J Am Coll Cardiol. 2005;45:1538-1542.

53. Tearney GJ, Regar E, Akasaka T, et al. Consensus standards for acquisition, measurement, and reporting of intravascular optical coherence tomography studies: a report from the International Working Group for Intravascular Optical Coherence Tomography Standardization and Validation. J Am Coll Cardiol. 2012;59:1058-1072.

54. Imamura T, Kinugawa K, Murasawa T, et al. Cardiac allograft vasculopathy can be distinguished from donor-transmitted coronary atherosclerosis by optical coherence tomography imaging in a heart transplantation recipient: double layered intimal thickness. Int Heart J. 2014;55:178-180.

55. Crespo-Leiro MG, Marzoa-Rivas R, Barge-Caballero E, Paniagua-Martin MJ. Prevention and treatment of coronary artery vasculopathy. Curr Opin Organ Transplant. 2012;17:546-550.

56. Kobashigawa JA, Katznelson S, Laks H, et al. Effect of pravastatin on outcomes after cardiac transplantation. New Engl J Med. 1995;333:621-627.

57. Wenke K, Meiser B, Thiery J, et al. Simvastatin reduces graft vessel disease and mortality after heart transplantation: a four-year randomized trial. Circulation. 1997;96:1398-1402.

58. Wenke K, Meiser B, Thiery J, et al. Simvastatin initiated early after heart transplantation: 8-year prospective experience. Circulation. 2003;107:93-97.

59. Kobashigawa JA, Moriguchi JD, Laks H, et al. Ten-year follow-up of a randomized trial of pravastatin in heart transplant patients. $J$ Heart Lung Transplant. 2005;24:1736-1740.

60. Mehra MR, Raval NY. Metaanalysis of statins and survival in de novo cardiac transplantation. Transplant Proc. 2004;36:1539-1541.

61. Erinc K, Yamani MH, Starling RC, et al. The effect of combined Angiotensin-converting enzyme inhibition and calcium antagonism on allograft coronary vasculopathy validated by intravascular ultrasound. J Heart Lung Transplant. 2005;24:1033-1038.
62. Snydman DR, Kistler KD, Ulsh P, Bergman GE, Vensak J, Morris J. The impact of CMV prevention on long-term recipient and graft survival in heart transplant recipients: analysis of the Scientific Registry of Transplant Recipients (SRTR) database. Clin Transplant. 2011;25:E455-E462.

63. Potena L, Grigioni F, Magnani G, et al. Prophylaxis versus preemptive anti-cytomegalovirus approach for prevention of allograft vasculopathy in heart transplant recipients. J Heart Lung Transplant. 2009;28:461-467.

64. Dasari TW, Hennebry TA, Hanna EB, Saucedo JF. Drug eluting versus bare metal stents in cardiac allograft vasculopathy: a systematic review of literature. Catheter Cardiovasc Interv. 2011;77:962-969.

65. Vigano M, Tuzcu M, Benza R, et al. Prevention of acute rejection and allograft vasculopathy by everolimus in cardiac transplants recipients: a 24-month analysis. J Heart Lung Transplant. 2007;26:584-592.

66. Topilsky Y, Hasin T, Raichlin E, et al. Sirolimus as primary immunosuppression attenuates allograft vasculopathy with improved late survival and decreased cardiac events after cardiac transplantation. Circulation. 2012;125:708-720.

67. Fang JC, Kinlay S, Beltrame J, et al. Effect of vitamins C and E on progression of transplant-associated arteriosclerosis: a randomised trial. Lancet. 2002;359:1108-1113.

68. Eckl S, Heim C, Abele-Ohl S, et al. Combination of clopidogrel and everolimus dramatically reduced the development of transplant arteriosclerosis in murine aortic allografts. Transpl Int. 2010;23:959-966.

69. Abele S, Weyand M, Wollin M, et al. Clopidogrel reduces the development of transplant arteriosclerosis. J Thorac Cardiovasc Surg. 2006;131:1161-1166.

70. Tsai VW, Cooper J, Garan H, et al. The efficacy of implantable cardioverter-defibrillators in heart transplant recipients: results from a multicenter registry. Circ Heart Fail. 2009;2:197-201.

71. Thajudeen A, Stecker EC, Shehata M, et al. Arrhythmias after heart transplantation: mechanisms and management. J Am Heart Assoc. 2012;1:1-8.

72. Aqel RA, Wells BJ, Hage FG, et al. Re-stenosis after drug-eluting stents in cardiac allograft vasculopathy. $J$ Heart Lung Transplant. 2008;27:610-615.

73. Agarwal S, Parashar A, Kapadia SR, et al. Long-term mortality after cardiac allograft vasculopathy: implications of percutaneous intervention. JACC Heart Fail. 2014;2:281-288.

74. Tjang YS, Tenderich G, Hornik L, et al. Cardiac retransplantation in adults: an evidence-based systematic review. Thorac Cardiovasc Surg. 2008;56:323-327.

75. Tsao L, Uriel N, Leitz K, et al. Higher rate of comorbidities after cardiac retransplantation contributes to decreased survival. J Heart Lung Transplant. 2009;28:1072-1074.

76. Arbustini E, Roberts WC. Morphologic observations in the epicardial coronary arteries and their surroundings late after cardiac transplantation (allograft vascular disease). Am J Cardiol. 1996;78(7):814-820.
Transplant Research and Risk Management

\section{Publish your work in this journal}

Transplant Research and Risk Management is an international, peerreviewed open access journal focusing on all aspects of transplantation and risk management to achieve optimal outcomes in the recipient improving survival and quality of life. The journal welcomes submitted papers covering original research, basic science, clinical studies,

\section{Dovepress}

reviews \& evaluations, guidelines, expert opinion and commentary, case reports and extended reports. The manuscript management system is completely online and includes a very quick and fair peer-review system, which is all easy to use. Visit http://www.dovepress.com/ testimonials.php to read real quotes from published authors. 